\title{
The various contributions to the diffuse scattering from PMN-xPT
}

D. Phelan ${ }^{1}$, M.J. Krogstad ${ }^{1}$, P.M. Gehring ${ }^{2}$, S. Rosenkranz ${ }^{1}$, R. Osborn ${ }^{1}$, F. Ye ${ }^{3}$, Y. Liu ${ }^{3}$, J.P.C. Ruff ${ }^{4}$, Z.-G. Ye ${ }^{5}$, and J.M. Wozniak ${ }^{6}$

${ }^{1}$ Materials Science Division, Argonne National Laboratory

${ }^{2}$ NIST Center for Neutron Research

${ }^{3}$ Quantum Condensed Matter Division, Oak Ridge National Laboratory

${ }^{4}$ CHESS

${ }^{5}$ Department of Chemistry, Simon Fraser University

${ }^{6}$ Mathematics and Computer Science Division, Argonne National Laboratory

Relaxor ferroelectrics, which have large electromechanical coupling, possess temperature and electric field dependent local atomic displacements that lead to diffuse patterns in diffraction experiments. Here, we discuss several different contributions to the diffuse scattering in the lead-based relaxor ferroelectrics, PMN-xPT. Insight into these contributions has been gained through measurements that employ state-of-the-art neutron and $x$-ray instrumentation allowing for efficient measurements of three-dimensional diffuse scattering over large wave-vector ranges and thus many Brillouin zones. Such measurements have been performed on a number of crystals which reveal the compositional dependence of the different features as the system morphs from a 'relaxor' character into a 'ferroelectric' character upon increasing $x$. 